\title{
USO DEL LÉXICO EN TEXTOS ESCRITOS POR ESTUDIANTES DE NIVEL B2 DE ESPAÑOL COMO SEGUNDA LENGUA
}

\author{
Ericka Vargas Castro
}

\section{(c) $(7)(9)$}

Esta obra está bajo una licencia Creative Commons 



\title{
USO DEL LÉXICO EN TEXTOS ESCRITOS POR ESTUDIANTES DE NIVEL B2 DE ESPAÑOL COMO SEGUNDA LENGUA
}

\author{
LEXICAL PERFORMANCE IN WRITINGS PRODUCED BY \\ STUDENTS OF SPANISH AS A SECOND LANGUAGE OF LEVEL B2 \\ Ericka Vargas Castro
}

\begin{abstract}
RESUMEN
El objetivo de este artículo es describir el uso del léxico en términos de variedad, propiedad, precisión y competencia en textos escritos por estudiantes con un nivel B2 de español como segunda lengua. Por un lado, interesa identificar los tipos de problemas más frecuentes en cuanto al empleo del vocabulario, pues a partir de estos se pueden diseñar actividades que ayuden a mejorar el desempeño de los alumnos. Por otro lado, se busca interpretar los resultados de acuerdo con la teoría de transferencia de lengua, para así determinar aquellas dificultades que evidencian necesidades específicas en torno al aprendizaje de lengua, y las que estarían relacionadas con el ámbito de escritura académica.

Palabras clave: léxico, transferencia de lengua, español como segunda lengua (ESL), aprendizaje de lenguas extranjeras, escritura académica.
\end{abstract}

\begin{abstract}
The aim of this paper is to describe the use of lexicon in terms of variety, property, accuracy, and competence in texts written by students of Spanish as a second language with level B2. First, we attempt to identify the most common types of problems regarding the use of vocabulary, because from them teachers can design activities to improve the performance of the learners. On the other hand, we seek to interpret the results according to language transfer theory to determine which difficulties effectively demonstrate specific needs regarding language learning and which ones would be related to the field of academic writing.

Key words: lexicon, language transfer, Spanish as a Second Language (SSL), foreign language learning, academic writing.
\end{abstract}

\section{Introducción}

Las redacciones escritas por estudiantes de español como segunda lengua (ESL) constituyen un recurso muy valioso para el docente. Muestran el producto final del proceso de escritura y, además, permiten identificar los aspectos lingüísticos sobre los cuales el alumno necesita trabajar para alcanzar el mejor desempeño posible en la lengua meta.

M. L. Ericka Vargas Castro. Universidad de Costa Rica. Profesora interina. Escuela de Filología, Lingüística y Literatura. Costa Rica.

Correo electrónico: erickavargasucr@gmail.com

Recepción: 30- 04- 2015

Aceptación: 01- 09- 2015 
Un estudiante que haya consolidado el nivel B2 destaca por el hecho de que ya ha pasado por un período prolongado de aprendizaje; por lo tanto, los problemas que presente en esta etapa deben trabajarse con particular atención para propiciar un verdadero acceso a los niveles que en el Marco Común Europeo de Referencia para las Lenguas (MCER) se denominan C1 (Dominio operativo eficaz) y C2 (Maestría), es decir, para que las destrezas lingüísticas logren ampliarse y el usuario pase de ser independiente a competente (Instituto Cervantes, 2012, p. 35).

Ahora bien, son muchos los aspectos que intervienen para producir un texto escrito: gramática, conectores discursivos, mecanismos referenciales, puntuación y léxico, entre otros. Esta mayor complejidad en términos de tiempo, de preparación y de evaluación ha sido una causa determinante para que los docentes se inclinen por enfatizar la lectura, la escucha y la conversación en clases de segunda lengua y releguen a un segundo plano las actividades relacionadas con escritura académica (Valdés, Haro y Echev-Arriarza, 1992, p. 333).

En el presente estudio, se realizará un análisis del desempeño léxico en términos de variedad, propiedad, precisión y competencia en escritos elaborados por estudiantes de ESL de nivel B2 para determinar, por un lado, las principales necesidades del alumnado en torno al vocabulario y, por otro lado, interpretarlas según el criterio conocido como transferencia de lengua. De este modo, se busca diferenciar las necesidades relacionadas con el aprendizaje de un segundo idioma de aquellas vinculadas con escritura académica y que, por ende, dependerían de las habilidades que se tengan para redactar en la lengua materna.

\section{Estudios relevantes sobre el léxico en español como segunda lengua}

Marconi (2000) y De Miguel-García (2005) afirman que las palabras son instrumentos que permiten desarrollar la competencia comunicativa porque no solo comprenden un nivel semántico, sino que además establecen relaciones referenciales, gramaticales, discursivas, socioculturales y le posibilitan al estudiante construir estrategias con el fin de llevar a cabo tareas determinadas con el lenguaje; de hecho, Singleton (1999, p. 1) señala que "almost everything in language is related in some way or other to words".

Por lo tanto, el vocabulario es fundamental para ayudar a determinar el nivel del alumno; incluso Cassany, Luna y Sanz (1998) mencionan que existen casos en los que se considera que se ha alcanzado un conocimiento avanzado a partir del número esperado de palabras que el aprendiz debería conocer. Es indudable que para medir el desempeño en una segunda lengua es necesario evaluar las producciones a partir de diversos componentes como gramática, fluidez, pronunciación, coherencia y cohesión, entre otros, pero el caudal y uso del léxico son de particular importancia porque propician la descripción clara y concisa de eventos y, además, muestran la habilidad del estudiante para desplazarse de un registro formal a uno informal o de un dialecto a otro.

Según Cassany, Luna y Sanz (1998), conocer una palabra implica: saber su pronunciación y ortografía, su flexión en género y número, sus distintas acepciones, y tener la habilidad de emplearla según se necesite en el contexto de comunicación, de acuerdo con criterios sociolingüísticos y dialectales. Estos autores destacan la diferencia entre el vocabulario pasivo, aquel cuyo significado se podría entender si se escucha o lee, y el activo, palabras que se emplean para hablar y escribir en la vida cotidiana.

Para Florenzano-Alcolea (2007, p. 2), el aprendizaje de vocabulario ha de ser concebido como un proceso cuantitativo que implica reorganizar el lexicón mental, y no se 
limita a la memorización de listas de palabras. Para que su adquisición resulte efectiva, es preciso que el término no solo sea utilizado a nivel oral, sino que también forme parte de los procesos de lecto-escritura; esto se debe a que, cuando las habilidades productivas se vinculan con las receptivas, es más fácil fijar y recordar los contenidos que se estudian. No obstante, es importante destacar que la oralidad responde a necesidades comunicativas inmediatas, mientras que la producción de redacciones en un segundo idioma es un proceso que toma más tiempo por su grado de complejidad.

\section{Fundamentación teórica}

El uso del léxico en la escritura académica debe cumplir con ciertas características como lo son: la variedad, es decir, evitar la repetición de palabras por medio de sinonimia, elisiones, hiperónimos y otros recursos; la propiedad, la cual implica realizar la selección léxica adecuada para transmitir lo que se quiere comunicar; y la precisión, que consiste en emplear términos específicos, de manera que se recomienda prescindir de aquellos cuyo significado es muy general (como cosa, algo y otros semejantes) para así propiciar que el escrito sea de fácil comprensión y su contenido unívoco. De hecho, los problemas más frecuentes detectados por Cassany (2009) en torno a la escritura académica se relacionan con repetición de palabras, el uso y abuso de expresiones fijas que funcionan como auténticas muletillas y los comodines, es decir, nombres, verbos o adjetivos con sentido muy genérico que se emplean a falta de otro término más específico (Cassany, 2009, p. 147).

Por su parte, el MCER (Instituto Cervantes, 2002, p. 108) define la competencia léxica como el conocimiento del vocabulario de una lengua y la capacidad para utilizarlo adecuadamente. En él se distingue entre aquellas palabras cuya función en el discurso es establecer relaciones gramaticales (artículos, cuantificadores, demostrativos, pronombres personales y relativos, así como adverbios interrogativos, posesivos, preposiciones, verbos auxiliares y conjunciones), y las de contenido semántico (sustantivos, adjetivos, verbos principales y adverbios). También se incluyen dentro del léxico el siguiente listado de expresiones hechas:

Cuadro 1. Competencia léxica (MCER, 2002, p. 108).

\begin{tabular}{|c|l|l|}
\hline Elemento & \multicolumn{1}{|c|}{ Definición } & \multicolumn{1}{|c|}{ Ejemplos } \\
\hline Fórmulas fijas & $\begin{array}{l}\text { Expresiones que siempre se usan en determinada situación } \\
\text { comunicativa, refranes, arcaísmos }\end{array}$ & $\begin{array}{l}\text { iBuenos días! } \\
\text { A mal tiempo } \\
\text { buena cara. }\end{array}$ \\
\hline Modismos & Metáforas lexicalizadas semánticamente opacas & $\begin{array}{l}\text { Estaba en las } \\
\text { nubes }\end{array}$ \\
\hline Estructuras fijas & $\begin{array}{l}\text { Estructuras a partir de las cuales se insertan frases para formar } \\
\text { Fraciones con determinado objetivo }\end{array}$ & $\begin{array}{l}\text { Por favor, } \\
\text { podría.. }\end{array}$ \\
\hline $\begin{array}{c}\text { Régimen } \\
\text { semántico }\end{array}$ & $\begin{array}{l}\text { Expresiones compuestas por palabras que habitualmente se } \\
\text { emplean juntas }\end{array}$ & $\begin{array}{l}\text { Soñar con } \\
\text { Por medio de }\end{array}$ \\
\hline
\end{tabular}


Asimismo, se brindan las siguientes escalas ilustrativas para la gradación del conocimiento del vocabulario:

Cuadro 2. Riqueza de vocabulario (Instituto Cervantes, 2002, p. 109).

\begin{tabular}{|c|l|}
\hline \multicolumn{2}{|c|}{ RIQUEZA DE VOCABULARIO } \\
\hline C2 & $\begin{array}{l}\text { Tiene un buen dominio de un repertorio léxico muy amplio que incluye expresiones idiomáticas y } \\
\text { coloquiales; muestra que es capaz de apreciar los niveles connotativos del significado. }\end{array}$ \\
\hline C1 & $\begin{array}{l}\text { Tiene un buen dominio de un amplio repertorio léxico que le permite superar con soltura sus } \\
\text { deficiencias mediante circunloquios; apenas se le nota que busca expresiones o que utiliza } \\
\text { estrategias de evitación. Buen dominio de expresiones idiomáticas y coloquiales. }\end{array}$ \\
\hline B2 & $\begin{array}{l}\text { Dispone de un amplio vocabulario sobre asuntos relativos a su especialidad y temas más generales. } \\
\text { Varía la formulación para evitar la frecuente repetición, pero las deficiencias léxicas todavía } \\
\text { pueden provocar vacilación y circunloquios. }\end{array}$ \\
\hline B1 & $\begin{array}{l}\text { Tiene suficiente vocabulario para expresarse con algún circunloquio sobre la mayoría de los temas } \\
\text { pertinentes para su vida diaria como, por ejemplo, familia, aficiones e intereses, trabajo, viajes y } \\
\text { hechos de actualidad. }\end{array}$ \\
\hline A2 & $\begin{array}{l}\text { Tiene suficiente vocabulario para desenvolverse en actividades habituales y en transacciones } \\
\text { cotidianas que comprenden situaciones y temas conocidos. } \\
\text { Tiene suficiente vocabulario para expresar necesidades comunicativas básicas. } \\
\text { Tiene suficiente vocabulario para satisfacer necesidades sencillas de supervivencia. }\end{array}$ \\
\hline A1 & Tiene un repertorio básico de palabras y frases aisladas relativas a situaciones concretas. \\
\hline
\end{tabular}

Cuadro 3. Dominio de vocabulario (Instituto Cervantes, 2002, p. 109).

\begin{tabular}{|l|l|}
\hline \multicolumn{2}{|c|}{ DOMINIO DE VOCABULARIO } \\
\hline C2 & Utiliza con consistencia un vocabulario correcto y apropiado. \\
\hline C1 & Pequeños y esporádicos deslices, pero sin errores importantes de vocabulario. \\
\hline B2 & $\begin{array}{l}\text { Su precisión léxica es generalmente alta, aunque tenga alguna confusión o cometa alguna } \\
\text { incorrección al seleccionar las palabras, sin que ello obstaculice la comunicación. }\end{array}$ \\
\hline B1 & $\begin{array}{l}\text { Manifiesta un buen dominio del vocabulario elemental, pero todavía comete errores } \\
\text { importantes cuando expresa pensamientos más complejos o cuando aborda temas y } \\
\text { situaciones poco frecuentes. }\end{array}$ \\
\hline A2 & Domina un limitado repertorio relativo a necesidades concretas y cotidianas. \\
\hline A1 & No hay descriptor disponible. \\
\hline
\end{tabular}

A partir de estos descriptores, puede notarse que, en un nivel B2, se esperaría un repertorio léxico amplio que involucre especificidad en torno a lo que se quiere transmitir, que sea variado y que se use de una forma apropiada según el contexto. Sin embargo, se reconoce el hecho de que pueden surgir problemas en la selección de los términos. Cabe destacar que, en el presente estudio, la competencia léxica se centrará en la dimensión lingüística (Jiménez-Catalán, 2002), específicamente en ortografía, morfología, colocaciones y restricciones sintácticas.

Ahora bien, cuando se opta por una composición como muestra para medir el desempeño léxico de un estudiante, hay que tener presente que adquirir destrezas en expresión escrita es un proceso lento, gradual y complejo y, por eso, conviene ser selectivo con los 
resultados. Redactar un texto formal con fines académicos implica planeamiento, organización de las ideas, elaboración del esquema, escritura y, por último, un periodo de revisión. De hecho, incluso los hablantes nativos que han tenido acceso a educación superior muestran problemas a la hora de plasmar sus ideas en un escrito (Sánchez-Avendaño, 2005, p. 218).

Además, es necesario tomar en cuenta el fenómeno conocido como transferencia de lengua, el cual consiste en extender los conocimientos previos y aplicarlos en un campo nuevo de aprendizaje (Solman y Chung, 1996, p. 174). Piedehierro (2009) emplea esta teoría para analizar la influencia de las experiencias anteriores del discente en la habilidad de escritura en una segunda lengua. En términos generales, se puede afirmar que las destrezas para redactar que una persona haya adquirido en su lengua materna se trasladan cuando aprende otro idioma. Por ejemplo, son transferibles las habilidades en cuanto a la planificación del escrito, la atención que se presta a la hora de organizar el texto, la capacidad para generar y mantener la coherencia y la efectividad con la que se enfrenta al proceso de revisión en la lengua extranjera (Cumming, 1989; Rocade-Larios y Murphy, 2001; Ferrari, 2004-2005) y, de acuerdo con los resultados de Piedehierro (2009), influye también la percepción que el estudiante tiene de lo que significa que un texto esté bien escrito y el tipo de instrucción que haya recibido. Esta capacidad de transferencia resulta crucial para interpretar las dificultades en torno al léxico que se manifiestan en las redacciones de los aprendices de español y así, a partir de ellas, brindar una retroalimentación más efectiva y fundamentada.

\section{Metodología}

Para realizar esta investigación, se recopilaron veinte redacciones escritas por estudiantes de ESL con un nivel B2. A los participantes se les solicitó leer un artículo sobre el sudoku y escribir un texto expositivo de 300 palabras en donde resumían el contenido dicha lectura y consideraban los aspectos positivos y negativos del empleo de este juego como técnica de ejercicio mental. A continuación, se procedió a identificar y clasificar los problemas relacionados con el léxico que aparecen en las producciones escritas en términos de variedad, propiedad, precisión y competencia. Posteriormente, se analizaron los resultados para determinar las principales necesidades léxicas del estudiantado en torno a la producción de escritos, así como en relación con su aprendizaje de lengua. Este análisis es ante todo de tipo cualitativo, pues interesa ahondar en las distintas selecciones léxicas que debilitan el texto aunque tengan poca incidencia; empero, se logró identificar aquellos desaciertos que se presentan en mayor medida.

\section{Resultados}

\subsection{Variedad}

La falta de variedad fue el problema de uso de léxico más frecuente; de hecho, debido a que en todas las producciones aquí analizadas se desarrolla el mismo tema, es posible determinar que las palabras que más se repitieron fueron: ejercicio, juego, cerebro, pasatiempo, ayuda y mente. La aparición de rubros léxicos iguales a lo largo del texto revela la ausencia de nuevas referencias para hacer mención a la misma entidad, las cuales se podrían propiciar con mecanismos como sinonimia, antonimia, hiperónimos, hipónimos, elipsis, uso apropiado 
de pronombres anafóricos y elisiones, entre otros. Asimismo, se registraron repeticiones de la misma raíz muy cercanas entre sí:

\begin{abstract}
1. Un estudio de NIH ha reflejado que los ejercicios de entrenamiento cognitivo, como el Sudoku, pueden mantener saludable el cerebro. El músculo "crece o se desarrolla" cuando se lo ejercita, dijo el experto, Guillermo Che Kenny. Estos ejercicios mentales estimulan al cerebro y también ayudan a retrasar la aparición del deterioro de la mente.
\end{abstract}

En este extracto se han empleado en dos ocasiones los sustantivos cerebro y ejercicio, así como de la raíz de este último término en ejercitar. Para Cassany (2009, p. 144), la repetición provoca monotonía y aburrimiento y es propia de escritores poco experimentados.

Si bien la falta de variedad muestra insuficiencias en cuanto al uso del léxico, esta no parece surgir exclusivamente a causa del desconocimiento de la lengua meta, pues dichas dificultades en la escritura también son frecuentes en hablantes nativos, según el estudio de Sánchez-Avendaño (2005). Este investigador se dedicó a analizar textos producidos por estudiantes del curso Básico de Redacción de la Universidad de Costa Rica, empleando como premisa teórica la linguística del texto. En el análisis de la coherencia, dicho autor identificó que los alumnos no recurren a los diversos mecanismos existentes para crear nuevas referencias sobre la misma entidad, sino que se limitan a repetir el mismo rubro léxico, sin variación alguna. Por lo tanto, problemas de este tipo tienen más conexión con deficiencias para generar escritos académicos que con necesidades particulares en el aprendizaje de lengua.

\title{
5.2 Propiedad
}

La categoría propiedad ocupa un nivel de frecuencia de aparición intermedio y es de particular interés, ya que aquí se registraron algunas dificultades relacionadas con el significado y uso de palabras:

\footnotetext{
2. Conozco un poco el tema porque los doctores dicen a mi bisabuela que su edad intercede con su memoria.

3. Probablemente, el partido de sudoku hace que una persona esté más alerta. Pero es difícil encontrar el tiempo jugar partidos mental.
}

En el ejemplo número dos, interceder, cuyo significado de acuerdo con la Real Academia Española (2012) es: "Hablar en favor de alguien para conseguirle un bien o librarlo de un mal", se ha utilizado en lugar del verbo interferir, lo cual pudo haber ocurrido por la similitud fonética entre ambos términos. Por otra parte, en el ejemplo número tres, hay una confusión entre los términos partido y juego, probablemente porque comparten contextos de aparición. Así, cuando se habla de fútbol se podrían escuchar enunciados como "el partido es en el estadio", "el juego empieza a las dos", "en el partido va a jugar Navas". No obstante, mientras todo partido consiste en la práctica de un deporte que busca competir en habilidades motrices con un rival, el juego es una actividad que se utiliza para la diversión y el disfrute de los participantes, por lo que podría decirse que todo partido es a su vez un juego, pero no todo juego es un partido.

Otro fenómeno que se relaciona con el ámbito léxico-semántico es el conocido como falsos amigos, definido en da Silva (2008) como dos vocablos idénticos o con mucha semejanza en términos gráficos y fonéticos cuyo significado diverge parcial o totalmente en dos lenguas dadas. A continuación se incorpora un enunciado que lo ejemplifica: 
4. Había muchos estudios sobre ese juego para soportar con hechos reales.

Se emplea aquí el verbo soportar, cuyo significado en español es "Sostener o llevar sobre sí una carga o peso" (RAE, 2012), con una acepción equivalente a apoyar, debido a la semejanza del primero con la voz inglesa 'support'. De acuerdo con Pérez-Velazco (2001), los falsos amigos deben ser considerados como errores sistemáticos, es decir, que se presentan con determinada frecuencia y que son, por lo tanto, predecibles.

Los hablantes tienden a cometer faltas en el nivel léxico por analogía, esto es, intentan producir una nueva forma, para ellos desconocida, tratando de imitar las ya existentes en su acervo lingüístico (Pérez-Velasco, 2001, p. 380). Hay errores de este tipo que son esperables en los niños cuya lengua materna es el español, por ejemplo, cuando dicen no cabo en lugar de no qиеро por presuponer un paradigma regular. Esta flexión desviada de la normativa evidencia que se encuentran en el proceso de adquisición de su lengua materna (L1). De la misma manera, los estudiantes extranjeros, cuando se encuentran en la necesidad de producir una palabra española de cuya forma no están seguros, tienden a recurrir a su L1, la cual les servirá como modelo. El empeño de Pérez Velasco por enfatizar el carácter recurrente de este tipo de errores radica en que, si se sabe que existe la posibilidad de que el aprendiz de español como segunda lengua los produzca, entonces es indispensable que el docente le brinde las herramientas lingüísticas para que logre suplir sus insuficiencias en torno al léxico de la mejor forma posible.

\subsection{Precisión}

Los resultados revelaron que la categoría en donde hubo menos problemas fue en precisión, y que las palabras con contenido general más empleadas fueron cosa y algo:

\footnotetext{
5. Es una cosa, todavía, muy popular en los Estados Unidos. No me gusta el Sudoku por una razón que es no me gustan las cosas que incluyen números o matemáticas.

6. Normalmente, este juego dura mucho tiempo, lo cual puede ser algo bueno o malo.
}

En el ejemplo número cinco, la palabra cosa empobrece el contenido y resta claridad al escrito debido a que su significado es muy general, de manera que obliga al lector a hacer un esfuerzo mayor para comprender los pensamientos del emisor. Una oración que indique que hay una cosa popular en los Estados Unidos no cumple con el principio de informatividad, el cual presupone partir de los elementos conocidos, para luego introducir lo que discursivamente se considera como nuevo, y así posibilitar la progresión temática (Sánchez-Avendaño, 2005). En este caso el lector no lograría delimitar con claridad en qué consiste la información nueva, porque existen gran cantidad de objetos que comparten la misma propiedad y que podrían ser incluidos dentro del lexema seleccionado (música, ropa, tecnología, entre muchas otras posibilidades). Asimismo, el empleo del mismo vocablo en la segunda oración no me gustan las cosas que incluyen números o matemáticas conduce a una multiplicidad de posibles interpretaciones: rechazo por los juegos en los que se utilizan números, apatía hacia las matemáticas como disciplina académica, o repulsión a toda actividad humana en donde se requiera el análisis matemático.

En el ejemplo seis, el alumno ha utilizado el término algo como un comodín, es decir, dicha palabra le permite rellenar provisionalmente un vacío léxico, pero lo hace con un elemento que debilita el contenido, por cuanto resultaría más apropiado sustituirla por un grupo nominal como una característica. Cassany (2009, p. 148) recomienda utilizar términos específicos, para que la idea del escritor sea clara y no surjan ambigüedades interpretativas. 
De acuerdo con Sánchez-Avendaño (2005, p. 286), palabras como cosa y algo son proformas léxicas que el hablante emplea para hacerle frente a sus necesidades comunicativas inmediatas, y son efectivas cuando hay un contexto compartido entre el emisor y el receptor, como ocurriría en conversaciones en las que los participantes están presentes. En esas circunstancias la vaguedad de los términos no dificulta el entendimiento pues la naturaleza del referente es clara. Empero, en un texto escrito no se cuenta con el apoyo del contexto compartido; por ende, la precisión del léxico empleado es clave para la adecuada decodificación del mensaje.

Tal como sucede con la falta de variedad, el uso de términos poco precisos es un aspecto recurrente en redacciones escritas por hablantes nativos de español, de manera que su empleo podría estar motivado por la carencia de criterios sobre cómo mejorar un texto escrito en la etapa de revisión, y no por deficiencias propias del proceso de aprendizaje de lengua.

\subsection{Competencia léxica}

Por último, en relación con la competencia léxica, se registraron problemas en la escritura de palabras, producto de la confusión entre la grafía inglesa y la española. Por ejemplo, 'necesito' apareció como necessito (similar a necessity) y 'famoso' presentó la diptongación ou (famouso). También se registró la forma stimulación 'estimulación', en donde se ha optado por una construcción que iría en contra de las reglas fonotácticas del español, las cuales rigen las secuencias posibles de fonemas (Azebedo, 2009, p. 94), pues este idioma no posee grupos consonánticos de tipo st como ataque de sílaba, sino que, cuando se ha aceptado una palabra extranjera que tiene esta estructura silábica dentro del léxico general de la lengua, se incorpora la vocal $e$ en posición inicial y esta forma un grupo silábico con la consonante siguiente.

Además, en las redacciones recopiladas se registraron algunos términos con un género que no les corresponde (las crucigramas, las rompecabezas) o que se utilizaron con una sufijación flexiva o derivativa inconsistente en relación con la categoría gramatical que exige el contexto (lee mucha, verdadera por verdaderamente).

La competencia léxica también se vio debilitada por requisitos gramaticales:

7. Mi pasatiempo favorito es solitario. No es tan difícil como el Sudoku, pero todavía ayuda a la mente y el cerebro porque tiene que pensar de todas las posibilidades del juego.

En la sección subrayada, se destaca una función sintáctica conocida por La Real Academia Española (2010, p. 685) como régimen preposicional, la cual se define como aquella que "desempeñan los grupos preposicionales argumentales, es decir, aquellos exigidos semánticamente por los predicados". La preposición que debe emplearse está determinada por razones tanto semánticas como históricas y no es predecible, por cuanto corresponde al estudiante familiarizarse con ella por medio del uso. En el segmento anterior, el discente combina el verbo pensar con la preposición de, cuando la unión debería darse con en.

Una dificultad que comparten los aprendices de ESL con los hablantes nativos surge cuando es preciso identificar las secuencias que requieren la preposición de frente a las que no:

8. Como se debe pensar mucho cuando se está haciendo el juego estoy seguro que ayuda al cerebro.

La estructura que el estudiante ha utilizado necesita la preposición, lo cual se puede verificar sustituyendo la cláusula subordinada por el pronombre determinante neutro eso (estoy seguro de eso), o bien construyendo la pregunta: ¿de qué estoy seguro?, pues la presencia de 
la preposición al inicio de la interrogante demuestra que su uso es requerido (Serafini, 2007).

En cuando a estructuras fijas, el problema principal detectado en las producciones analizadas radica en la confusión que genera la sintaxis requerida por verbos como gustar (Complemento Indirecto + Verbo + Sujeto) frente a otros que se vinculan con un orden Sujeto + Verbo + Objeto. Lo anterior se manifiesta con verbos cuyo significado también denota preferencias, como en a mí odio este juego (por odio este juego). Lo que podría estar ocurriendo es que, al conocer la existencia de algunos otros verbos que siguen la estructura de gustar (como me fascina, me encanta, me parece bien, etc.), se genera duda sobre cuáles tendrán un CI al inicio de la oración y cuáles un sujeto. Este tipo de producción agramatical también se formó con opinar: A mí me opina que las escuelas necesitan usar estos ejercicios en sus planes de estudios. Dicha debilidad, en principio sintáctica, se extiende al nivel léxico porque afecta la flexión verbal en expresiones utilizadas para transmitir opiniones personales y puntos de vista.

Otro de los puntos sobre léxico que destaca el MCER es el de relaciones semánticas, esto es, las colocaciones. Moreno-Pereiro, Buyse, Hogeschool, y Leuven (2003, p. 10) las definen como "combinaciones frecuentes de unidades léxicas, pero en su uso más restringido se trata de combinaciones características de dos lexemas entre los que existe cierta afinidad". Los autores brindan una serie de características para poder identificar las colocaciones en un sentido estricto, entre las cuales figuran: la coocurrencia significativamente frecuente de dos lexemas, la relación típica entre sus componentes, cierta flexibilidad formal y su significado literal. Entonces, un ejemplo de colocación sería tocar la guitarra, pues en español se ha seleccionado ese verbo para designar la acción de hacer sonar artísticamente un instrumento.

Las colocaciones resultan ser un reto para los estudiantes de ESL porque, en muchas ocasiones, en su lengua materna se presenta una tendencia de combinación diferente de la que se emplea en castellano. Por ejemplo, Molero y Salazar (2013) comentan que la expresión dar un paseo se traduce al inglés como take a walk (verbo tomar), en italiano como fare una passeggiata (verbo hacer), en alemán como einen Spaziergang machen (verbo hacer) y en polaco como pójść na spacer (verbo ir).

En las redacciones del corpus, aparecieron las siguientes combinaciones:

\footnotetext{
9. Con los estudios los investigadores tienen de acuerdo la importancia de hacer ejercicio.

10. Hay muchas maneras de hacer ejercicio mental y el artículo da atención al sudoku, que es popular en Japón y los EE. UU.

11. Mantener la salud del cerebro es muy importante para mí, entonces voy a gastar más tiempo en realizar más pasatiempos como sudoku y otros así
}

En el primer caso, el verbo tener se ha combinado con el grupo preposicional de acuerdo, cuando la colocación esperada en español sería con estar o ponerse. En el segundo ejemplo, se combina dar con atención, cuando la selección más adecuada la proporcionarían los verbos poner o prestar. En el tercer enunciado, la colocación para tiempo en el sentido propuesto por el estudiante debería ser pasar, en cuyo caso se generaría la repetición pasar tiempo en pasatiempos, por cuanto se recomendaría reformular la oración.

En este apartado la dimensión sociolingüística de la competencia léxica no resultó relevante, ya que no se rastrearon usos que estuvieran directamente relacionados con un dialecto o un registro en particular. Por el contrario, el conocimiento lingüístico del uso de una palabra emerge cada vez que el estudiante quiere transmitir una idea, pues tendrá que vincular las palabras entre sí, de manera que se torna evidente cualquier selección gráfica/fonética, 
morfológica, sintáctica o semántica que no se adapte con las estructuras propias del español. Esta categoría es de particular interés para los docentes, en vista de que evidencia el grado de dominio de la L2.

\section{Conclusiones y recomendaciones}

La variedad, propiedad y precisión son tres aspectos claves en el uso del léxico en escritura académica. Aunque en los tres ámbitos se mostraron deficiencias en las redacciones en estudio, se pudo verificar que el de la variedad aparece en mayor medida, mientras que las dificultades en torno a la precisión fueron mucho menores. Ahora bien, en vista de que estos problemas también son comunes en hablantes nativos, puede concluirse que su aparición no es evidencia fehaciente de que el estudiante carece del vocabulario necesario para expresar sus ideas, sino que puede obedecer a la necesidad de mayor instrucción y práctica en el ámbito de escritura académica.

En relación con la propiedad, se demostró la necesidad de ampliar el conocimiento léxico del estudiante a través de actividades que involucren términos con sinonimia parcial, o bien con cierta gradación semántica, para así lograr que los aprendices sean más sensibles a los diversos matices de significado que brindan algunas palabras. También se mostró que una debilidad recurrente son los falsos amigos, los cuales se consideran errores cuya presencia durante el aprendizaje de una lengua es altamente esperable y, por ello, merecen atención en una clase de L2. Estos pueden estudiarse a través de textos, a partir de los cuales los discentes identifiquen si los términos están utilizándose adecuadamente según el contexto.

En cuanto a la competencia léxica, las deficiencias principales giraron en torno al régimen preposicional, estructuras fijas y colocaciones. Es importante recordar que a nivel de psicolingüística, estas últimas se procesan como una sola unidad (Zimmer, 2010). El diario vivir está lleno de situaciones ritualizadas y contextos que generan respuestas lingüísticas específicas, muchas de ellas determinadas culturalmente (Dinnen, 1995). Por lo tanto, la eficacia de enseñar colocaciones y frases hechas radica en que el aprendiz estaría adquiriendo destrezas pragmáticas para crear efectos específicos en intercambios de la vida real. La competencia léxica evidencia el conocimiento lingüístico de las propiedades morfosintácticas de una palabra, por cuanto es fundamental ejercitarla en las clases de segunda lengua.

Por otra parte, las reflexiones que se han generado gracias a este estudio permiten concluir que las dificultades en cuanto al léxico en las redacciones de estudiantes de una L2 deben ser analizadas e interpretadas según los objetivos del curso. Si el propósito principal es la enseñanza/aprendizaje de lengua, resulta relevante centrarse en la propiedad, así como en la competencia léxica. Es recomendable que, en los niveles B y C, los aspectos que se desean mejorar se trabajen a nivel discursivo y tomando en cuenta situaciones comunicativas reales, es decir, extractos de lenguaje utilizados por sus hablantes en la cotidianeidad, para que el alumno pueda deducir cómo se van combinando las palabras entre sí, y qué implicaciones lingüísticas, sociales y pragmáticas conlleva su empleo.

En cambio, si el curso está orientado a la enseñanza de escritura académica para estudiantes de ESL, se deben tomar en cuenta todos los puntos que se consideraron en esta investigación, por cuanto es recomendable, por un lado, dedicar tiempo al análisis del uso del léxico en contexto, matices de significado, relaciones fijas entre palabras y su adaptación según aspectos sociolingüísticos y dialectales y, por otro lado, repasar los diversos aspectos que intervienen en el 
proceso de revisión de un texto escrito. En este punto correspondería identificar las repeticiones, para luego proceder a emplear diversos mecanismos cohesivos con miras a evitarlas y, además, sería necesario verificar que no haya palabras comodines y, si las hubiese, reemplazarlas por términos específicos cuyo significado sea pertinente de acuerdo con el objetivo comunicativo.

En resumen, no se puede asumir en una clase de ESL que la enseñanza de la lengua dará como resultado un desempeño léxico óptimo en redacciones, ya que las habilidades que se tengan en escritura académica dependerán de las experiencias previas del estudiante, o bien de los nuevos conocimientos sobre redacción que se vayan adquiriendo. Por ende, la manera en la que se interpretan y evalúan las debilidades en torno al léxico se deben centrar en medir el grado de eficacia con el que se han asimilado los contenidos específicos del curso.

\section{Bibliografía}

Azebedo, M. (2009). Introducción a la lingüística española. New Jersey: Prentice Hall.

Cassany, D., Luna, M. y Sanz, G. (1998). Enseñar lengua. Barcelona: Graó.

Cassany, D. (2009). La cocina de la escritura. (16 ed.). Barcelona: Anagrama.

Cumming, A. (1989). Writing Expertise and Second-Language Proficiency. Language Learning. 39 (1), 81-135.

Da Silva, J. (2008). Los significados de los falsos amigos: español/portugués. Revista Letra Magna. 4 (8), 1-11. http://www.letramagna.com/falsosamigos.pdf/ [Consulta 22 de abril de 2015].

De Miguel-García, M.L. (2005). La enseñanza del léxico del español como lengua extranjera. Resultados de una encuesta sobre la metodología aplicada en el aula. Revista de Didáctica Marco ELE. 1, 1-21. http://marcoele.com/descargas/1/de_miguel-lexico-ele. pdf/ [Consulta 22 de abril de 2015].

Dinnen, F. (1995). General Linguistics. Washington: Georgetown University Press.

Ferrari, V. (2004-2005). Transferencia de estrategias de escritura de lengua materna a lengua extranjera. Lenguas Modernas. 30, 123-159.

Florenzano-Alcolea, M. (2007). El tratamiento del vocabulario en el aula de español para inmigrantes. Revista Electrónica de Didáctica ELE. 10, 26-55. http:// www.mecd.gob.es/dctm/redele/Material-RedEle/Revista/2007_10/2007_ redELE_10_03Florenzano.pdf?documentId=0901e72b80df3076. [Consulta 22 de abril de 2015].

Instituto Cervantes. (2002). Marco Común Europeo de Referencia para las Lenguas. http:// www.forem.es/assets/files/mcerl.pdf/ [Consulta 15 de noviembre de 2014].

Jiménez-Catalán, R. (2002). El concepto de competencia léxica en estudios de aprendizaje y enseñanza de segundas lenguas. Atlantis. 24 (2), 149-162.

Marconi, D. (2000). La competencia léxica. Madrid: Antonio Machado Libros.

Molero, C. M., y Salazar, D. (2013). Análisis contrastivo, criterios de selección y didáctica de las colocaciones léxicas en el aula de español. Por B. Pregelj (Ed.). Estudios 
Hispánicos en el siglo XXI. Belgrado: Universidad de Belgrado. http://www.fil. bg.ac.rs/wp-content/uploads/obavestenja/iberijske/ehes21/22Molero,\%20Salazar.pdf/ [Consulta 15 de noviembre de 2014].

Moreno-Pereiro. S., Buyse, K., Hogeschool, L., y Leuven, K.U. (2003). Colocaciones léxicas: pistas y trampas. Revista Mosaico, Consejería de Educación en Bélgica. 10, 1-9. http:// wwwling.arts.kuleuven.be/elektravoc/bestanden/colocaciones.pdf/ [Consulta $17 \mathrm{de}$ noviembre de 2014].

Pérez-Velasco, J. M. (2001). Los falsos amigos: adquisición de lenguas y cambio lingüístico. Por I. Uzcanga-Vivar, E. Llamas-Pombo, y J. M. Pérez-Velasco (Eds.). Presencia y renovación de la lingüística francesa. (377-384). Salamanca: Ediciones de la Universidad de Salamanca.

Piedehierro, C. (2009). La transferencia de instrucción. Un rasgo de la interlengua de aprendices francófonos de ELE. Revista Nebrija de Lingüística Aplicada a la Enseñanza de Lenguas. 5 (3), 55-86.

Real Academia Española [RAE]. (2010). Manual de la nueva gramática de la lengua española. México D.F.: Editorial Planeta Mexicana.

Real Academia Española [RAE]. (2012). Diccionario de la lengua española. http://www.rae. es/recursos/diccionarios/drae. [Consulta 22 de abril de 2015].

Roca-de-Larios, J., y Murphy, L. (2001). Some Steps Towards a Socio-cognitive Interpretation of Second Language Composition Processes. International Journal of English Studies. 1 (2), 25-45.

Sánchez-Avendaño, C. (2005). Los problemas de redacción de los estudiantes costarricenses: una propuesta de revisión desde la lingüística del texto. Revista de Filología y Lingüística. 31 (1), 267-295.

Serafini, M. T. (2007). Cómo se escribe. Barcelona: Paidós.

Singleton, D. (1999). Language and Lexicon: An Introduction. California: Routledge.

Solman, R., y Chung, K. (1996). Language Transfer and blocking in Second Language Vocabulary Learning. Journal of Behavioral Education. 6 (2), 173-190.

Valdés, G., Haro, P., y Echev-Arriarza, M. P. (1992). The Development of Writing Abilities in a Foreign Language: Contributions toward a General Theory of L2 Writing. The Modern Language Journal. 76 (3), 333-352.

Zimmer, B. (2010, 16 de setiembre). Chunking. The New York Times Magazine. http://www. nytimes.com/2010/09/19/magazine/19FOB-OnLanguage-Zimmer.html/ [Consulta 15 de diciembre de 2014]. 